\title{
Fast calculation of the Lomb-Scargle periodogram using nonequispaced fast Fourier transforms
}

\author{
B. Leroy \\ LESIA, Observatoire de Paris, CNRS, UPMC, Université Paris-Diderot, 5 place Jules Janssen, 92195 Meudon, France \\ e-mail: Bernard.Leroy@obspm.fr \\ Received 20 February 2012 / Accepted 23 July 2012 \\ ABSTRACT

\begin{abstract}
We present a code for the fast computation of the Lomb-Scargle periodogram that uses nonequispaced fast Fourier transforms (FFTs). The computation time has the classical $O(N \log N)$ behaviour of FFTs, but is shorter by about one order of magnitude than the "classical" fast algorithm by Press and Rybicki, and is about five times shorter that the best GPU-based implementations of the usual $O\left(N^{2}\right)$ algorithm. This performance is achieved without sacrificing accuracy, as revealed by comparing the computations done with our FFT-based algorithm and a naïve one.
\end{abstract}

Key words. methods: data analysis - methods: numerical - stars: oscillations

\section{Introduction}

The lightcurves obtained with spatial missions such as CoRoT (Michel et al. 2008) and Kepler (Koch et al. 2010) generally consist of extremely large numbers of samples (typically several hundreds of thousands). In addition, these long timesseries cannot be evenly sampled over their full duration (e.g., because of the passage of the spacecraft through radiation belts, or because some CCD pixels may be dead, etc.). As a result, the search for periods in the lightcurves cannot be made with direct recourse to the fast Fourier transform (FFT).

In the presence of a nonuniformly sampled lightcurve, it is not uncommon to transform it by interpolation into one with a uniform sampling before doing spectral analysis via FFT. This, however, is not entirely satisfactory since it modifies the statistical properties of the data by introducing artificial data.

One of the most popular alternatives is the accelerated computation of the Lomb-Scargle periodogram proposed by Press \& Rybicki (1989), which dramatically improves, in terms of running time, on the original periodogram developed by Lomb (1976) and Scargle (1982). Interestingly, the basic idea behind the Lomb-Scargle periodogram was originally presented in Gottlieb et al. (1975). Though this results in the evaluation of an approximation to the periodogram, it has been generally adopted not only because it is fast, but also because it retains a good accuracy. Another approach was proposed by Townsend (2010), where instead of evaluating an approximation, the exact periodogram is evaluated by leveraging the computing power of graphics processing units (GPUs). In spite of relying on a $O\left(N^{2}\right)$ algorithm (where $N$ is the number of samples), this runs on a state-of-the art GPU and is slightly faster than the "classical" $O(N \log N)$ algorithm by Press \& Rybicki on a conventional processor (CPU).

In this paper, we propose to evaluate an approximation to the Lomb-Scargle periodogram by taking advantage of comparatively recent algorithms developed for the fast calculation of
Fourier transforms of nonuniformly sampled times-series. Thus, we use the software library developped by Keiner et al. (2009), namely the library of nonequispaced FFTs (NFFTs). The computational time scales with the number of data samples, $N$, practically as $O(N \log N)$ (for the details, see Keiner et al. 2009); however, the implied constant in the $O$-notation is smaller than that of Press \& Rybicki mainly owing to the use the state-of-theart FFT implementation in the FFTW library (Frigo \& Johnson 2005). The accuracy of the NFFT is excellent (Steidl 1998).

The paper is organised as follows. In Sect. 2, we briefly recall the formalism of the Lomb-Scargle periodogram. Section 3 introduces the general ideas behind the NFFT algorithms. A code for computing an NFFT-based periodogram is presented in Sect. 4. Benchmarking computations and comparisons with existing codes are presented in Sect. 5. Finally, several perspectives are outlined in Sect. 6.

\section{The Lomb-Scargle periodogram}

Here we give a brief overview of the formalism of the LombScargle periodogram. Given a set of $N$ measurements $y_{i}$ at observation times $t_{i}(i=1, \ldots, N)$, the Lomb-Scargle normalised periodogram at frequency $f$ is defined as (Press \& Rybicki 1989)

$$
\begin{aligned}
P_{N}(f)= & \frac{1}{2 \sigma^{2}}\left\{\frac{\left[\sum_{i}\left(y_{i}-\bar{y}\right) \cos \omega\left(t_{i}-\tau\right)\right]^{2}}{\sum_{i} \cos ^{2} \omega\left(t_{i}-\tau\right)}\right. \\
& \left.+\frac{\left[\sum_{i}\left(y_{i}-\bar{y}\right) \cos \omega\left(t_{i}-\tau\right)\right]^{2}}{\sum_{i} \cos ^{2} \omega\left(t_{i}-\tau\right)}\right\},
\end{aligned}
$$

where $\omega=2 \pi f, \bar{y}$, and $\sigma^{2}$ are the mean and variance of the measurements, respectively, given by

$\bar{y}=\frac{1}{N} \sum_{i} y_{i}, \sigma^{2}=\frac{1}{N-1} \sum_{i}\left(y_{i}-\bar{y}\right)^{2}$, 
and the time-offset $\tau$ is defined by

$\tan 2 \omega \tau=\frac{\sum_{i} \sin 2 \omega t}{\sum_{i} \cos 2 \omega t}$

Here and throughout this section, the summations run from $i=1$ to $i=N$.

Scargle (1982) and Schwarzenberg-Czerny (1998) investigated the statistical distribution of the periodogram under the assumption that the measurements originate from pure Gaussian white noise. Schwarzenberg-Czerny (1998) showed that, in this case, a periodogram, normalised by the total variance as in Eq. (1), follows a Beta distribution $I_{x}[1,(N-3) / 2]$, defined as

$I_{x}(a, b)=\frac{\mathrm{B}_{x}(a, b)}{\mathrm{B}(a, b)}$,

with $x=2 P_{N}(f) / N$, and where $\mathrm{B}(a, b)$ and $\mathrm{B}_{x}(a, b)$ are, respectively, the Beta function and the incomplete version thereof, defined as (NIST 2011)

$\mathrm{B}_{x}(a, b)=\int_{0}^{x} t^{a-1}(1-t)^{b-1} \mathrm{~d} t$

In the case of Gaussian white noise, the false-alarm probability that a periodogram comprising $M$ independent frequencies will exhibit a peak due to chance fluctuations is

$$
\begin{aligned}
P_{\mathrm{FA}} & =1-\left[1-I_{x}\left(1, \frac{N-3}{2}\right)\right]^{M} \\
& =1-\left[1-\left(1-\frac{2 P_{N}(f)}{N}\right)^{(N-3) / 2}\right]^{M} .
\end{aligned}
$$

The non-trivial question of whether we can determine the number of independent frequencies in a periodogram has been addressed in the literature (Horne \& Baliunas 1986; Frescura et al. 2008), and is not considered here because that would be beyond the scope of this paper.

As noted by Press \& Rybicki (1989), the sums that occur in Eq. (1) can be greatly simplified. If we define

$$
\begin{array}{ll}
S_{y}=\sum_{i}\left(y_{i}-\bar{y}\right) \sin \omega t_{i}, & C_{y}=\sum_{i}\left(y_{i}-\bar{y}\right) \cos \omega t_{i}, \\
S_{2}=\sum_{i} \sin 2 \omega t_{i}, & C_{2}=\sum_{i} \cos 2 \omega t_{i},
\end{array}
$$

then

$$
\begin{aligned}
& \sum_{i}\left(y_{i}-\bar{y}\right) \cos \omega\left(t_{i}-\tau\right)=C_{y} \cos \omega \tau+S_{y} \sin \omega \tau, \\
& \sum_{i}\left(y_{i}-\bar{y}\right) \sin \omega\left(t_{i}-\tau\right)=S_{y} \cos \omega \tau-C_{y} \sin \omega \tau, \\
& \sum_{i} \cos ^{2} \omega\left(t_{i}-\tau\right)=\frac{N}{2}+\frac{1}{2} C_{2} \cos 2 \omega \tau+\frac{1}{2} S_{2} \sin 2 \omega \tau, \\
& \sum_{i} \sin ^{2} \omega\left(t_{i}-\tau\right)=\frac{N}{2}-\frac{1}{2} C_{2} \cos 2 \omega \tau-\frac{1}{2} S_{2} \sin 2 \omega \tau .
\end{aligned}
$$

These simplifications form the basis of the code proposed in Press et al. (1992). These authors emphasised that if the $t_{i}$ were evenly spaced, the above sums could be evaluated by two complex FFTs before substituting them back into Eqs. (1) and (3). To be able to use FFTs despite the nonuniformity of the $t_{i}$, they invented the so-called extirpolation process, which is based on the Lagrange interpolation (see their paper for details). Here, we instead propose to bypass the extirpolation process, and to directly compute the sums given in Eq. (8) by using complex NFFTs, since a new numerical tool to evaluate these now exists under the form of a freely available, high-quality library written in C language (Keiner et al. 2009).

\section{The NFFT algorithm and library}

We present the principles of the computation of an NFFT, to ensure that the library implementing it does not appear as a mere black box, and that motivated users will be able to understand and adjust the fine tunings offered by the library.

If a continuous signal $y(t)$ is sampled every $\Delta t$ seconds at $N$ observation times $t_{j}=j \Delta t \quad(j=0, \ldots, N-1)$, a time series $y_{j}=y\left(t_{j}\right)$ is obtained. Its discrete Fourier transform is defined as

$Y_{k}=\sum_{j=0}^{N-1} y_{j} \mathrm{e}^{-2 \mathrm{i} \pi j k / N} \quad(k=-N / 2, \ldots, N / 2-1)$.

The domain of $k$ is written so as to reflect that there are only $N$ independent discrete frequencies $f_{k}=k /(N \Delta t)$, and that these satisfy

$-f_{c} \leq f_{k}<f c$,

where $f_{c}=1 /(2 \Delta t)$ is the Nyquist frequency. The inverse transform is

$y_{j}=\frac{1}{N} \sum_{k=-N / 2}^{N / 2-1} Y_{k} \mathrm{e}^{2 \mathrm{i} \pi j k / N} \quad(j=0, \ldots, N-1)$.

The mathematicians who developed the NFFT (Keiner et al. 2009) define the nonequispaced discrete Fourier transform (NDFT) as the generalisation of Eq. (11) without the normalising constant

$y\left(x_{j}\right)=\sum_{k=-N / 2}^{N / 2-1} Y_{k} \mathrm{e}^{-2 \mathrm{i} \pi k x_{j}} \quad(j=0, \ldots, M-1)$,

where the complex quantities $Y_{k}$ are given, $x_{j}$, are reduced times, called nodes, satisfying $-1 / 2 \leq x_{j}<1 / 2$, and one does not necessarily have $M=N$. (If $N$ is odd, the summation runs from $-[N / 2]$ to $[N / 2]$, where the brackets mean the integer part.) This can be written in matrix-vector notation as

$\boldsymbol{y}=\mathbf{A} Y$,

where A is a complex $M \times N$ matrix $\left(A_{j k}=\mathrm{e}^{-2 \mathrm{i} \pi k x_{j}}\right)$. This matrix is generally not square, and had it been so, it would generally be neither orthogonal nor invertible. The definition of the inverse NDFT is therefore a complicated issue. It is, instead, customary to consider the adjoint NDFT defined by

$\boldsymbol{Z}=\mathbf{A}^{\mathrm{H}} \boldsymbol{y}$,

where $\mathbf{A}^{\mathrm{H}}$ is the adjoint (or conjugate transpose) of matrix $\mathbf{A}$. This corresponds to the sums

$Z_{k}=\sum_{j=0}^{M-1} y\left(x_{j}\right) \mathrm{e}^{2 \mathrm{i} \pi k x_{j}} \quad(k=-N / 2, \ldots, N / 2-1)$, 
which is the customary formulation needed for the computation of a periodogram.

In view of the duality expressed by Eqs. (13) and (14), it turns out that the same methods can be applied to compute the sums in Eqs. (12) and (15).

The NFFT is an efficient computation of the NDFT defined in Eq. (12). To this end, the trigonometric polynomial

$y(x)=\sum_{k=-N / 2}^{N / 2-1} Y_{k} \mathrm{e}^{-2 \mathrm{i} \pi k x}$

is first approximated by suitable linear combinations of translates of a window function $\varphi$ having good localization in time/space and frequency ${ }^{1}$. The function $y(x)$ in Eq. (16) is periodic and has a period equal to 1 , therefore the function $\varphi(x)$, too, is chosen to be 1-periodic. Introducing an oversampling factor $\sigma>1$ and setting $n=\sigma N, y(x)$ is approximated by

$s_{1}(x)=\sum_{l=-n / 2}^{n / 2-1} g_{l} \varphi(x-l / n)$.

The question is then to define $g_{l}$ such that $s_{1} \approx y$. Converting the right-hand side of Eq. (17) to the frequency domain yields

$s_{1}(x)=\sum_{k \in \mathbb{Z}} G_{k} \Phi(k) \mathrm{e}^{-2 \mathrm{i} \pi k x}$

where

$G_{k}=\sum_{l=-n / 2}^{n / 2-1} g_{l} \mathrm{e}^{2 \mathrm{i} \pi k l / n}$

and

$\Phi(k)=\int_{-1 / 2}^{1 / 2} \varphi(x) \mathrm{e}^{2 \mathrm{i} \pi k x} \mathrm{~d} x \quad(k \in \mathbb{Z})$.

If $\Phi(k)$ becomes sufficiently small for $|k|>n / 2$ and if $\Phi(k) \neq 0$ for $-n / 2 \leq k<n / 2$, then comparison of Eqs. (16) and (18) suggests that one defines $\hat{g}_{k}$ such that

$G_{k}= \begin{cases}0, & k=-n / 2, \ldots,-N / 2-1, \\ f_{k} / \Phi(k), & k=-N / 2, \ldots, N / 2-1, \\ 0, & k=N / 2, \ldots, n / 2-1 .\end{cases}$

From Eq. (19), $g_{l}$ is now given by

$g_{l}=\frac{1}{n} \sum_{k=-N / 2}^{N / 2-1} \mathrm{e}^{-2 \mathrm{i} \pi k l / n} \quad(l=-n / 2, \ldots, n / 2-1)$,

which can be computed by an FFT of length $n$. If $\varphi$ is also localised in the time domain, so that it can be approximated by a 1-periodic function $\psi$

$\psi(x)=\varphi(x) \chi_{[-m / n, m / n]}(x) \quad(m<n, m \in \mathbb{N})$,

where $m$ is a cutoff parameter and $\chi_{[-m / n, m / n]}(x)$ is the characteristic function defined as

$\chi_{[-m / n, m / n]}(x)= \begin{cases}1, & -m / n \leq x \leq m / n, \\ 0, & \text { otherwise, }\end{cases}$

\footnotetext{
1 The approximation of a function by translates of a basis function is a well-known method in approximation theory (e.g. Schaback 1995).
}

then an approximation to $s_{1}$ is defined by

$s\left(x_{j}\right)=\sum_{l=-n / 2}^{n / 2-1} g_{l} \psi\left(x_{j}-l / n\right)=\sum_{l=\left[n x_{j}\right]-m}^{\left[n x_{j}\right]+m} g_{l} \psi\left(x_{j}-l / n\right)$.

To summarise, one has

$y\left(x_{j}\right) \approx s_{1}\left(x_{j}\right) \approx s\left(x_{j}\right)$,

which translates into the following three-step algorithm. Given a suitably chosen window function $\varphi$, we (i) compute $G_{k}$ according to Eq. (21); (ii) compute the Fourier transform of $G_{k}$ using FFT, which is efficiently achieved with the help of the FFTW library (Frigo \& Johnson 2005); (iii) compute the sum $s\left(x_{j}\right)$ of Eq. (25). Each of these three steps is linear and can be conveniently represented by a matrix (respectively, $\mathbf{D}, \mathbf{F}$, and $\mathbf{B}$ ), so that Eq. (13) reads

$\boldsymbol{y}=\mathbf{A Y} \approx \mathrm{BFDY}$.

As noted earlier, for the computation of a periodogram we actually need the adjoint NFFT, i.e.,

$\boldsymbol{Z}=\mathbf{A}^{\mathrm{H}} \boldsymbol{y} \approx \mathbf{D}^{\mathrm{T}} \mathbf{F}^{\mathrm{H}} \mathbf{B}^{\mathrm{T}} \boldsymbol{y}$.

This is essentially the same algorithm taken in reverse order, therefore its performances are the same as that for the direct NFFT. In particular, the arithmetical complexity of the algorithm can be shown to be $O(N \log N+M)$ (Keiner et al. 2009).

In addition, we note that the two approximations in Eq. (26) introduce, respectively, an aliasing error and a truncature error, both of which could be rigorously analysed for various window functions (e.g. Potts et al. 1998). This contrasts with several of the earlier NFFT algorithms.

The above NFFT algorithm and its adjoint are efficiently implemented in the NFFT library (Keiner et al. 2009), written in $\mathrm{C}$ language and freely downloadable ${ }^{2}$. This library is not limited to one-dimensional Fourier transforms that are nonequispaced in time, and provides a large bestiary of transforms in several dimensions; in particular, the case nonequispaced in both time and frequency is considered. The library also provides iterative methods to compute the corresponding inverse transforms. Moreover, several of the more important window functions considered in the literature are implemented, and their parameters have default values that yield smaller aliasing and truncating errors.

\section{An NFFT-based Lomb-Scargle periodogram code}

We now present NFFTLS, a code for computing a Lomb-Scargle periodogram that is a straightforward implementation of Eq. (1) rewritten in terms of the sums in Eq. (8) computed using NFFTs.

The periodogram is output on a uniform frequency grid

$f_{k}=k \Delta f \quad\left(k=1, \ldots, N_{f}\right)$,

where the frequency spacing is given by

$\Delta f=\frac{1}{C_{\text {over }}\left(t_{N_{t}}-t_{1}\right)}$,

${ }^{2}$ See http://www-user.tu-chemnitz.de/ potts/nfft/ 
$C_{\text {over }}$ is an oversampling parameter ${ }^{3}$, and the number of frequencies is

$N_{f}=\frac{1}{2} C_{\text {over }} C_{\text {high }} N_{t}$.

The control parameter $C_{\text {high }}$ is the ratio of the highest desired frequency, $f_{\text {high }}$, to the Nyquist frequency, $f_{c}=N_{t} /(2 T)$, that would be obtained if the $N_{t}$ data points were evenly sampled over the total sampling time $T=t_{N_{t}}-t_{1}$. The oversampling parameter and the high frequency parameter are both user inputs. It should be emphasised that in practice the choice of these parameters must be guided by the data sampling and the scientific problem at hand.

As mentioned in Sect. 3, the NFFT library assumes that the time range is the interval $[-1 / 2,1 / 2)$; therefore, before the computation of the NFFTs, the original time range must be mapped to this interval. This is achieved by applying the following transformation

$t_{i} \rightarrow x_{i}=2 a\left(t_{i}-t_{1}\right) \Delta f-a$,

with $a=\frac{1}{2}-\epsilon$, where $\epsilon$ is an arbitrarily small number (typically $\left.10^{-5}\right)$.

Figure A.1 lists the C99 code for the computation of the Fourier transform of an unevenly sampled times-series with the help of the NFFT library. We note that, owing to the implementation of the library, not the direct transform (Eq. (12)), but the adjoint transform (Eq. (15)) must be computed. Moreover, we note that the Fourier transform is not normalised. Though the function computes the transform at all frequencies $-m \delta f \leq f \leq m \delta f$ (with $m=N_{f}$ ), it outputs only the positive frequency part, since only strictly positive frequencies are involved in the computation of the periodogram.

For the computation of the transforms in Eq. (7) at angular frequencies $2 \omega$, it suffices to compute a Fourier transform of the observational window (i.e., with the data samples equal to 1.0) that is twice as long as that of the data

$W_{k}=\sum_{j=0}^{M-1} \mathrm{e}^{2 \mathrm{i} \pi k x_{j}} \quad(k=-N, \ldots, N-1)$,

and to take every second Fourier component. It turns out that the extra calculation that needs to be made negligly affects the time performances of the code.

The outline of the program is then very simple:

1. Read the input data (times-series).

2. Compute the mean and the variance of the data.

3. Centre the data around their mean.

4. Reduce the time span to the interval $[-1 / 2,1 / 2)$, taking oversampling into account (Eq. (32)).

5. Determine the maximum frequency element to be output.

6. Compute the Fourier transform of the data (Eq. (15) expressed in NFFT terms as Eq. (28)).

7. Compute the Fourier transform (of double length) of the window (Eq. (33) expressed as Eq. (28) where $\boldsymbol{y}$ has all its components equal to unity).

8. Compute the sums of Eq. (8), then the periodogram (Eq. (1)).

9. Output the periodogram.

10. Output false-alarm probability (Eq. (6)) vs. frequency (optional).

\footnotetext{
3 Note that this oversampling parameter has nothing to do with the "internal" parameter of the same name used in the NFFT approximation.
}

We note that steps 5 to 8 are actually wrapped into a $\mathrm{C}$ function that returns a $\mathrm{C}$-structure containing the two arrays that make up the periodogram, viz. the frequencies and the corresponding periodogram ordinates. Figure A.2 lists the code of that function.

\section{Tests and comparisons with existing codes}

The tests were conducted on two platforms. One is a laptop Dell Latitude E6400 that runs 64-bit Ubuntu GNU/Linux, with 3 GB of RAM. It has an Intel(R) Core(TM)2 Duo P9700 Processor, with a cache size of $6 \mathrm{MB}$ and a clock speed of $2.8 \mathrm{GHz}$. The other one is a 64-bit Debian GNU/Linux server with 47 GB of RAM. It has four hexa-core Intel(R) Xeon(R) Processors X5650, each with a cache size of $12 \mathrm{MB}$ and a clock speed of $2.66 \mathrm{GHz}$. No attempt was made to parallelise the code, and the program was run on only one core of a single chip. Hence, as the clock speeds of both platforms are quite similar, the running times of the same program executed on them essentially differ because of the effects of the cache and RAM sizes. The executables were created on both machines with the GNU gcc compiler (v4.6.1 for the laptop and v4.4.5 for the server), using the standard C99 language (option $-\mathrm{std}=\mathrm{c} 99$ ) and the -02 optimization flag.

For the validation of our code NFFTLs, we used several sets of simulated data, regularly as well as irregularly sampled, some with gaps. We compared the results with a code based on a straightforward implementation of the DFT, which is hence very slow because it is based on a $O\left(N^{2}\right)$ algorithm. The maximal absolute deviation between the results of both codes was found to be of the order of $10^{-8}$. In addition, the presence of gaps in the data or a random sampling did not alter the performances of NFFTLS, in terms of neither runtime nor numerical accuracy.

For the performance comparisons, we used the following codes: FASPER, the fast algorithm proposed by Press \& Rybicki (1989), in its C version (Press et al. 1992), and culsP the GPUbased algorithm proposed by Townsend (2010).

One should be aware that the GPU-based algorithm, culsP, is a straightforward implementation of exact expressions for the Fourier transforms, whilst FASPER and NFFTLS are both implementations of approximate expressions thereof (reverse interpolation or extirpolation in FASPER, and those summarised by Eq. (26), and described in Sect. 3, in NFFTLS) in order to take advantage of the FFT.

To compare the performances of our code to Townsend's CuLsP 2010, we used the same validation data set, namely the 150-day photometric time-series of the large-amplitude $\beta$ Cephei pulsator V1449 Aql (HD 180642) obtained by the CoRoT mission $^{4}$ (Belkacem et al. 2009). After removal of the flux measurements flagged as bad, the times-series comprised 382003 points, unevenly sampled. Whilst the sampling of the data set is mostly regular (with a sampling interval of about $32 \mathrm{~s}$ ), there are gaps of various sizes (see Fig. 1).

The periodogram of V1449 Aql was computed with NFFTLS. Figure 2 (upper plot) displays the part surrounding the peak corresponding to the dominant $0.18 \mathrm{~d}$ pulsation period (Waelkens et al. 1998). To get an idea of the accuracy, we also computed the periodogram using an implementation of the DFT that is a straightforward translation of Eq. (15). Figure 3 shows a scatter plot of the straightforward DFT- and NFFT-based periodograms against one another over the full frequency range. The good

\footnotetext{
4 The CoRoT space mission, launched on 2006 December 27, was developed and is operated by the CNES, with participation of the Science Programs of ESA, ESA's RSSD, Austria, Belgium, Brazil, Germany, and Spain.
} 


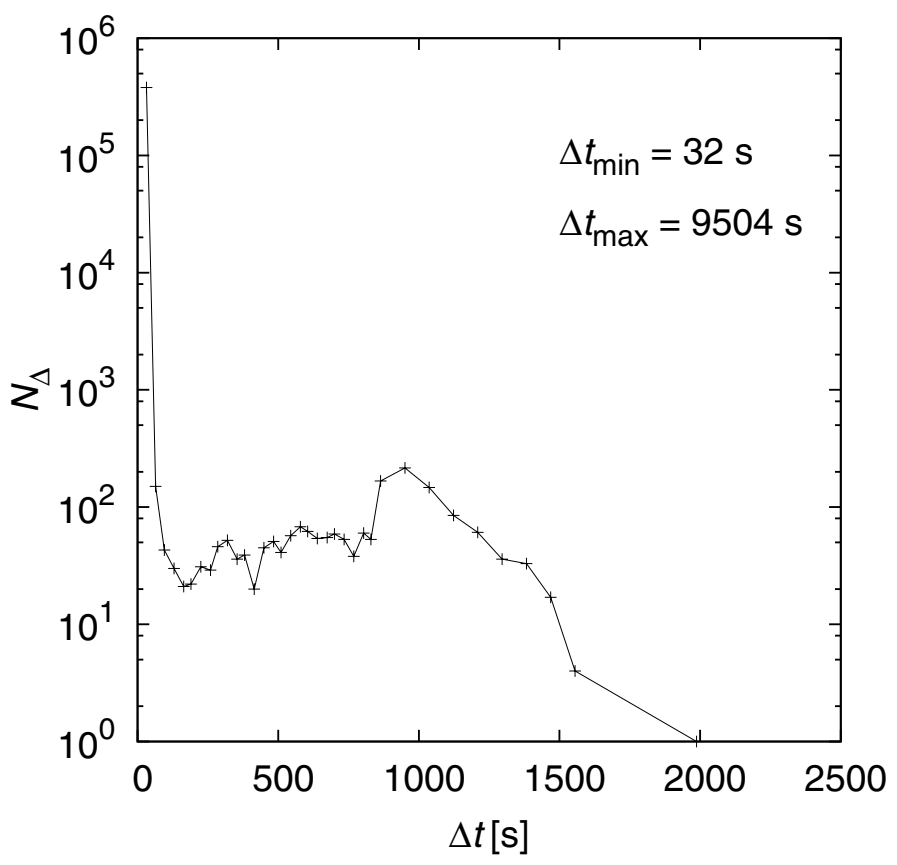

Fig. 1. Histogram of the sampling intervals in the V1149 Aql data set. The number of sampling intervals, $N_{\Delta}$, of a given value, $\Delta t$, is plotted only up to $\Delta t=2000 \mathrm{~s}$; the maximal sampling interval is a huge gap of $\Delta t_{\max }=9504 \mathrm{~s}$. The minimal sampling interval $\Delta t_{\min }=32 \mathrm{~s}$ corresponds to the nominal observation mode.
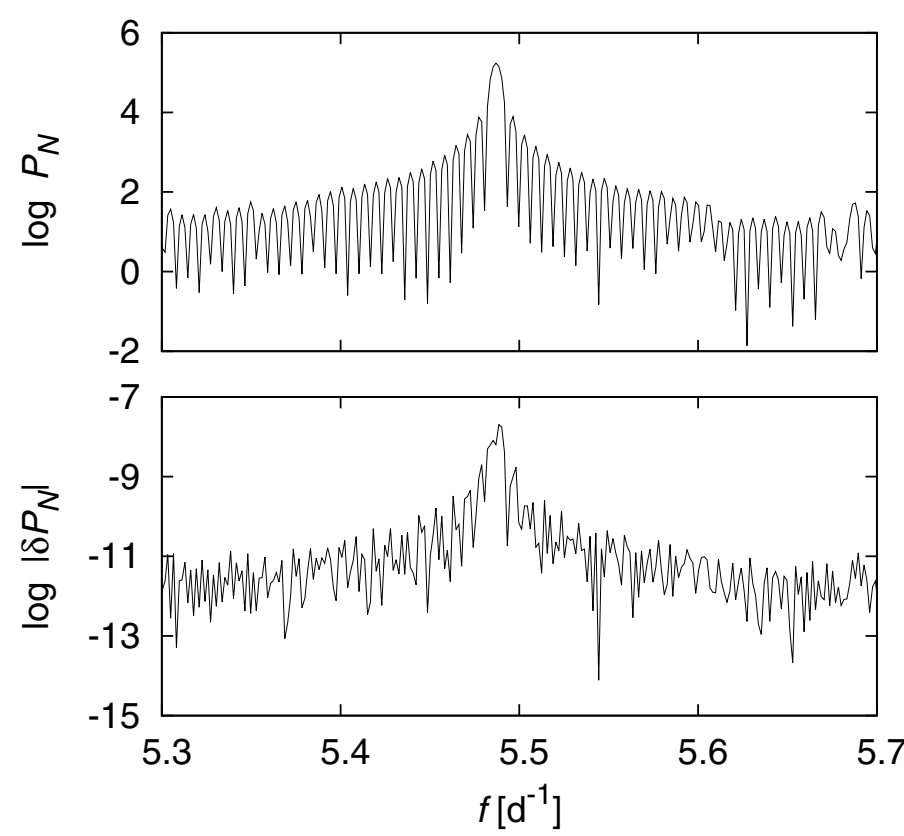

Fig. 2. Upper plot: part of the L-S periodogram for V1449 Aql; lower plot: absolute deviation $\left|P_{N}^{\mathrm{NFFT}}-P_{N}^{\mathrm{DFT}}\right|$ of the corresponding periodogram evaluated with an ordinary DFT code. Compare this figure with Fig. 2 of Townsend (2010).

agreement between both codes is clear: no point in the plot departs from the diagonal line $P_{N}^{\mathrm{NFFT}}=P_{N}^{\mathrm{DFT}}$, the maximal absolute "error" is $\max _{0<f \leq f_{\max }}\left|P_{N}^{\mathrm{NFFT}}-P_{N}^{\mathrm{DFT}}\right| \sim 2.0 \times 10^{-8}$ (see lower plot in Fig. 2), and the corresponding relative "error" is of the order of $10^{-13}$. This illustrates that the approximation performed

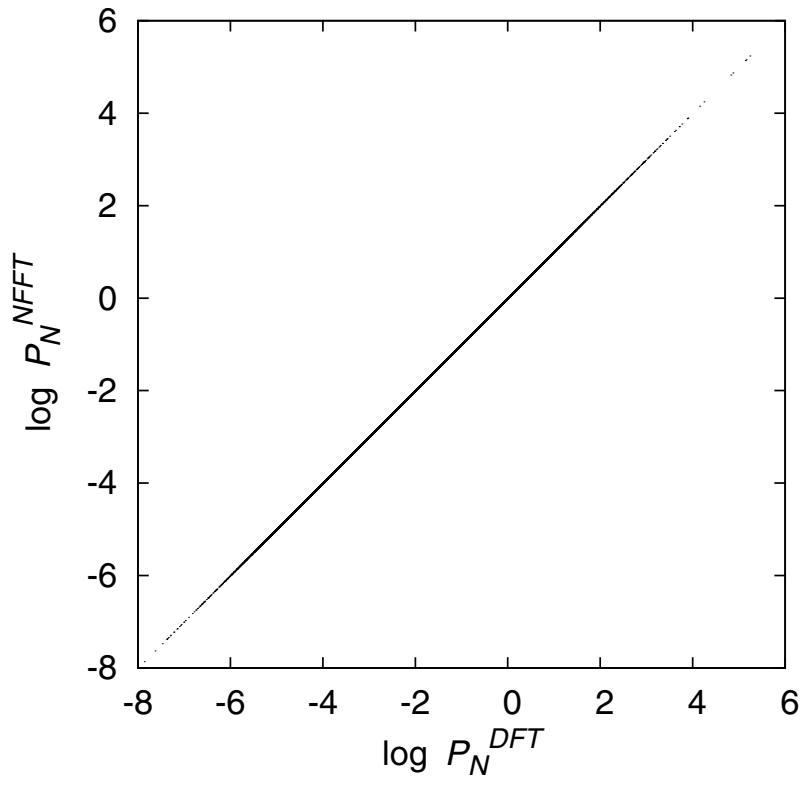

Fig. 3. Scatter plot of the NFFT-based periodogram against the straightforward DFT-based periodogram for V1449 Aql.

Table 1. Periodogram computation times in seconds.

\begin{tabular}{llcl}
\hline \hline Code & Platform & $\left\langle t_{\text {calc }}\right\rangle$ & $\sigma\left(t_{\text {calc }}\right)$ \\
\hline \multicolumn{4}{c}{ CPU implementation } \\
NFFTLS & Dell Laptop & 5.6 & 0.057 \\
NFFTLS & Server & 5.1 & 0.039 \\
FASPER & Dell Laptop & 42. & 1.1 \\
FASPER & Server & 30. & 1.4 \\
& \multicolumn{2}{c}{ GPU implementation } \\
CULSP & GeForce 8400 GS & 570. & 0.0093 \\
CULSP & Tesla C1060 & 20.3 & 0.00024 \\
\hline
\end{tabular}

Notes. For comparison, we have listed the computation times of culsP, the GPU implemented code by Townsend (2010), as well as a code based on the function FASPER of Press et al. (1992). We used an oversampling of 8 and a high-frequency control factor of 1 , which yields a periodogram with $N_{f}=1528012$ points, about the same number as the 1528064 points used in the runs of culsP.

by the NFFT-based code in computing a periodogram is extremely good. We note that a relative error of about that same size is measured when comparing the absolute value of a DFT computed without recourse to the FFT and one computed by using the NFFT library. All computations were done in double precision.

To measure the performance of NFFTLS and ease the comparison, we adopted Townsend's approach. We averaged the periodogram computation time over 100 runs (the typical running time for one computation is so short, that repetition was necessary). To make a fair comparison, we took into consideration only the time necessary to execute steps 5 to 8 (inclusive) of the code outline given in Sect. 4 . Table 1 lists the mean periodogram computation time $\left\langle t_{\text {calc }}\right\rangle$ and the corresponding standard deviation $\sigma\left(t_{\text {calc }}\right)$ for NFFTLS running on both the laptop and the server; for comparison, we also list the performances of a 
A\&A 545, A50 (2012)

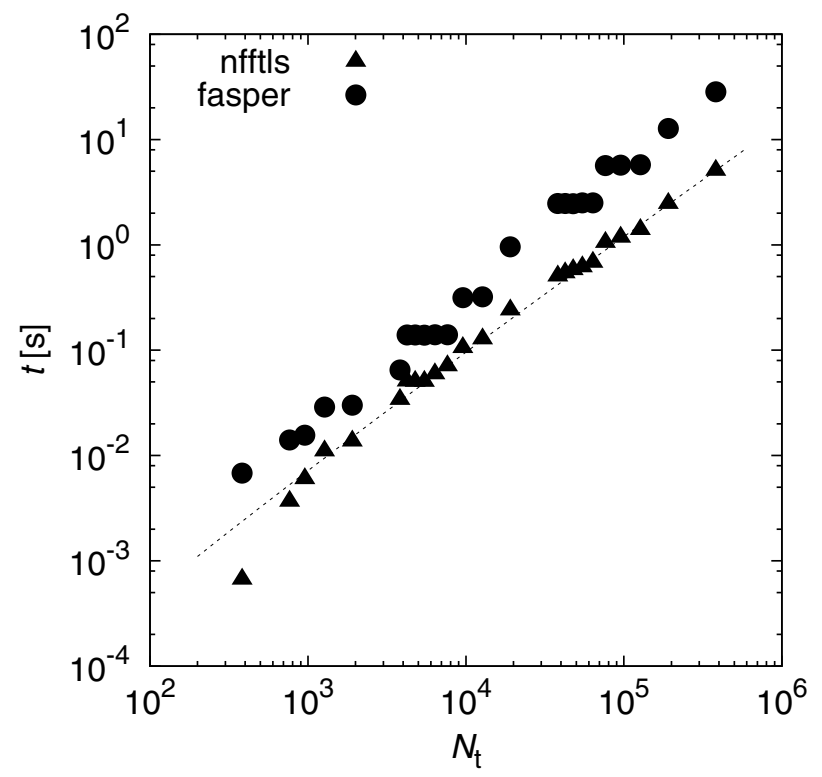

Fig. 4. Running time as a function of the length of the times-series of FASPER and NFFTLS. The dotted straight line is a least squares fit with a function proportional to $N_{t} \log N_{t}$.

code based on FASPER and Townsend's code CULSP. We modified the function FASPER by Press et al. (1992) by introducing instructions for timing the code at places most similar to those they have in NFFTLS, so that the comparison of running times was as fair as possible. In addition, we note that the computations in FASPER are done essentially in single precision, whilst they are done in double precision in NFFLTS. The use of single precision generally seriously impairs the accuracy, and depending on the computer architecture, the running time may also be affected ${ }^{5}$. We note that if the running time values for NFFTLS are corrected by adding the time necessary for executing steps 2 to step 4 , this amounts to increasing the corresponding tabulated values by $\sim 8 \mathrm{~ms}$, which is negligible.

Townsend's platform has a clock frequency of $2.33 \mathrm{GHz}$, which is not very different from that of our platforms $(2.67 \mathrm{GHz}$ and $2.80 \mathrm{GHz}$ ), therefore one may infer that the running times of NFFTLS and FASPER on his platform would be comparable to that displayed in Table 1 (the RAM size of Townsend's platform is $8 \mathrm{~GB}$, hence bracketed by the RAM sizes of our platforms).

Figure 4 plots the running time of FASPER and NFFTLS as a function of the length $N_{t}$ of the times-series. The times-series for a given $N_{t}$ is obtained by downsampling the original one. Although both are $O\left(N_{t} \log N_{t}\right)$ codes, clearly, the latter runs faster, viz. at least about six times faster, as indicated in Table 1. This difference in performance may be ascribed in large part to the implementation of the FFT on which both codes rest, and in particular to the use of the FFTW library in computing NFFTs.

In addition, NFFLs outperforms the GPU-based codes, typically running about four times faster than a code running on a Tesla C1060, one of the upper-end GPUs available on the market. Moreover, the computation with GPUs are still limited to a single precision accuracy, whilst codes running on CPUs can benefit from the built-in double precision of the standard $\mathrm{C}$ libraries. This presumably explains the great difference in the relative errors registered by NFFTLS and culsP. As far as the com-

\footnotetext{
5 In $\mathrm{C}$, this generally results in an increase in running time since the mathematical libraries for this language are in double precision.
}

putation of periodogram ordinates is concerned, this should not be too great a problem since the relative errors are very small in both cases. In this context, the most important considerations are the good performances in terms of running time.

Finally, we note that we found no improvement could be achieved by changing the default settings of the NFFT library, namely by departing from the Kaiser-Bessel window function (Jackson et al. 1991; Fourmont 1999) and/or by modifying default values of the parameters that control the quality of the NFFT approximation (viz. the "internal" oversampling and the cutoff parameter mentioned in Sect. 3).

\section{Conclusions and perspectives}

We have shown that the use of NNFTs could significantly shorten the running time for computing the periodogram of nonuniformly sampled light curves whilst ensuring an excellent accuracy. This, of course, assumes that the libraries used provide state-of-the-art implementations of the FFT and of the NFFTs. In this respect, the quality of the treatment of the approximations done by Keiner et al. (2009) in computing NFFTs makes their library highly commendable.

One should be aware that, for the mass treatment of the many stars for which space missions are now providing very long lightcurves, even a gain in running time of about one order of magnitude, as we obtain, over the "classical" accelerated algorithm by Press \& Rybicki (1989) is a valuable improvement. In addition, not only periodogram-orientated programs but virtually any spectral analysis program that must deal with irregularly sampled data could benefit from using NFFTs.

For example, Scargle (1989) discusses in detail Fourier transforms and correlation functions of irregularly sampled data, but provides an implementation of the DFT based on a $O\left(N^{2}\right)$ algorithm, which is therefore virtually impractical for very long times-series. Nothing in his discussion, however, prevents the replacement of his implementation with an NFFT, which would result in a dramatic decrease in running time. To this end, the implementation given in Fig. A.1 may serve as a guide. At this point, we wish to mention that the inversion back to the time domain performed by taking the FFT of a DFT evaluated at evenly spaced frequencies, as proposed by Scargle, might be replaced with the use of one of the iterative solvers proposed by Keiner et al. (2009) in their NFFT library; typically, a solver iteratively solves, e.g., Eq. (13) for $\boldsymbol{Y}$ given $\boldsymbol{y}$.

Among the various programs that may greatly benefit from the use of NFFTs, we only mention the one that is most used in stellar astronomy: SIGSPEC by Reegen (2007). Though an extremely effective tool, it is terribly slow because it does not make any recourse to the use of FFTs. Unfortunately, the complexity of the source code and its author's death make the corresponding adaptation a challenging (though certainly worthwhile) task.

In view of the already very good performances reached in computing an NFFT-based periodogram on a single processor core, it will be interesting to investigate whether parallelism may bring further improvement. We note that there have been some recent developments (Pippig 2009) towards producing a parallel version of the NFFT library ${ }^{6}$. However, we find that the available software remains insufficiently robust for it to serve as a basis for a code delivered to a large community.

\footnotetext{
${ }^{6}$ See http://www-user.tu-chemnitz.de/ mpip/software/
} 
The general trends in computer technology is not only towards multicore CPUs but also towards programmable GPUs. In addtion, computations of NFFTs on GPUs have started to appear (Sørensen et al. 2008). However, GPU computing remains too hardware-dependent, and monothread CPU computing is much easier to program ${ }^{7}$.

We therefore believe that the excellent performances that can be obtained with monothread computing using high-quality libraries, such as the FFTW and NFFT libraries, should continue to make codes such as NFFTLS extremely attractive.

\section{Appendix A: C-code for computing a periodogram}

In this appendix, we introduce the two building blocks necessary for computing a normalised Lomb-Scargle periodogram by using NFFTs. The complete program, NFFTLs, is freely available, under the GNU General Public License (GPLv3) ${ }^{8}$.

Figure A.1 presents the function, NFFT, that computes the NFFT of a times-series (the so-called dirty spectrum) or the corresponding observational window. To compute the latter, a NULL pointer must be provided as the argument for the measurements.

If the appropriate flags in the nnft_plan are set after it has been initialised (line 26), an optimisation is obtained by precomputing the values of $\hat{\varphi}(k)$ for $k=-N / 2, \ldots, / N 2-1$ (Eq. (20)). The library's default settings are such that the test on line 46 evaluates to true, so that another optimisation is done by precomputing (line 47) the values of $\psi\left(x_{j}-l / n\right)$ for $j=1, \ldots, M$ and $l=\left[n x_{j}\right]-m, \ldots,\left[n x_{j}\right]+m$ (Eq. (25)). We tried to improve the performance by varying these flags, but did not find any significant difference in the code performance. The library's defaults seem to conform perfectly to our needs. For more details on the available optimisations, the reader is referred to Keiner et al. (2009).

Figure A.2 presents the function periodogram used to compute the periodogram proper. Before the call, the user must have centred the measurements around their mean, and the observation times must have been reduced so as to lie in the interval $[-1 / 2,1 / 2)$. The function returns the results in a C-structure, $\mathrm{LS}$, defined as

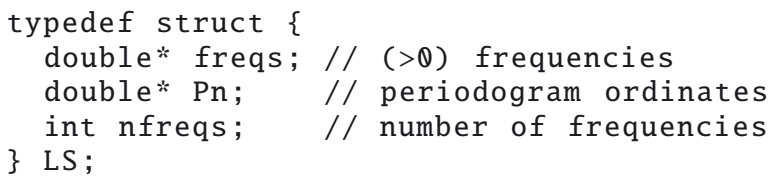

whose members are labelled freqs and Pn are two dynamically allocated arrays of size nfreqs. This structure is allocated by the function itself (lines 26-29); it is however the responsibility of the user to free the corresponding memory.

The undisplayed header file declar.h (line 3) contains the declarations of the function $\mathrm{nfft}$ as well as of the auxiliary functions

- sign (line 48), which transfers the sign of its second argument to its first $\operatorname{argument}$, i.e. $\operatorname{sign}(\mathrm{a}, \mathrm{b}) \operatorname{returns}|a| \operatorname{sgn} b$, where $\operatorname{sgn}(x)$ yields the sign of $x$;

\footnotetext{
${ }^{7}$ But this situation may change in a near future, as Intel recently (Aug. 2011) announced they are porting their Cilk Plus $\mathrm{C} / \mathrm{C}++$ language extensions for multithreaded parallel computing into the GNU Compiler collection (http://software.intel.com/en-us/ articles/intel-cilk-plus-open-source/).

${ }^{8}$ See http://corot-be.obspm.fr/
}

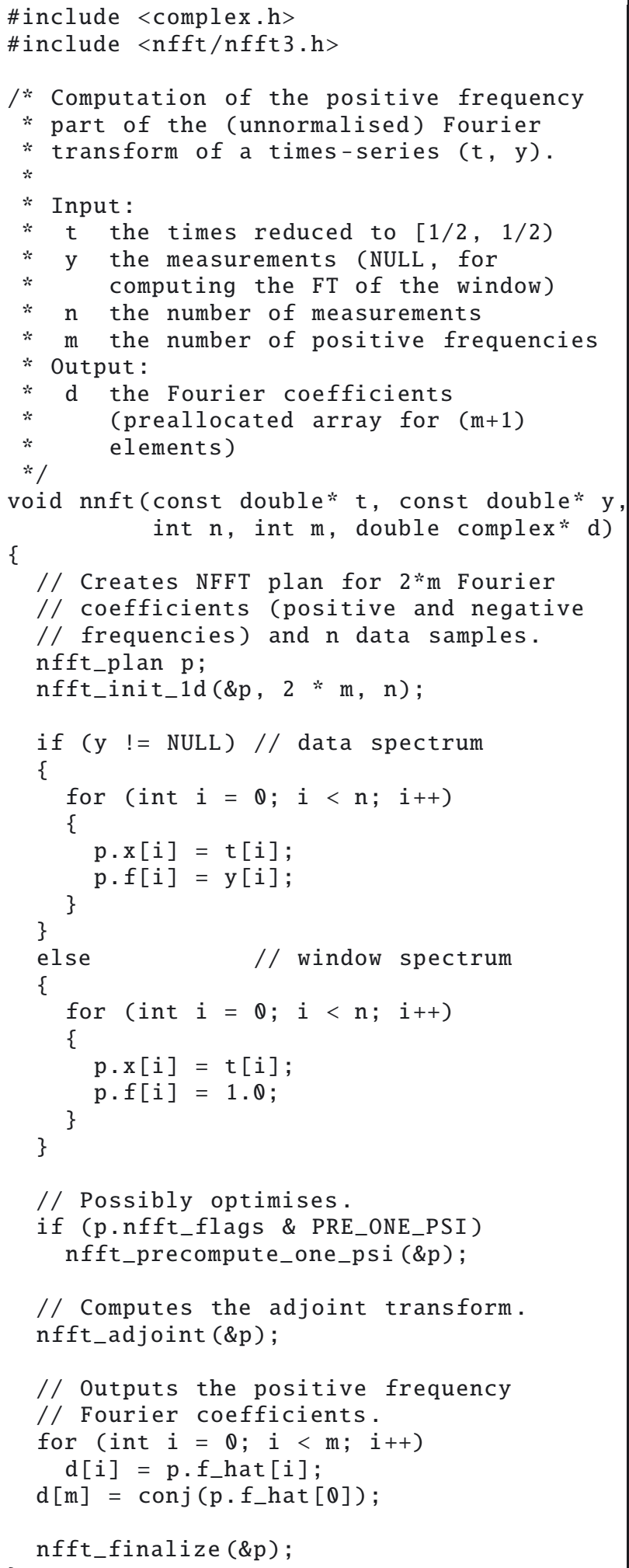

Fig. A.1. Function for computing an NFFT.

- square (lines 50-51), which returns the square of its argument;

- xmalloc (lines 26-28, 32, 35), which is a wrapper of the standard function malloc that terminates the program with a message in the case of an error. 


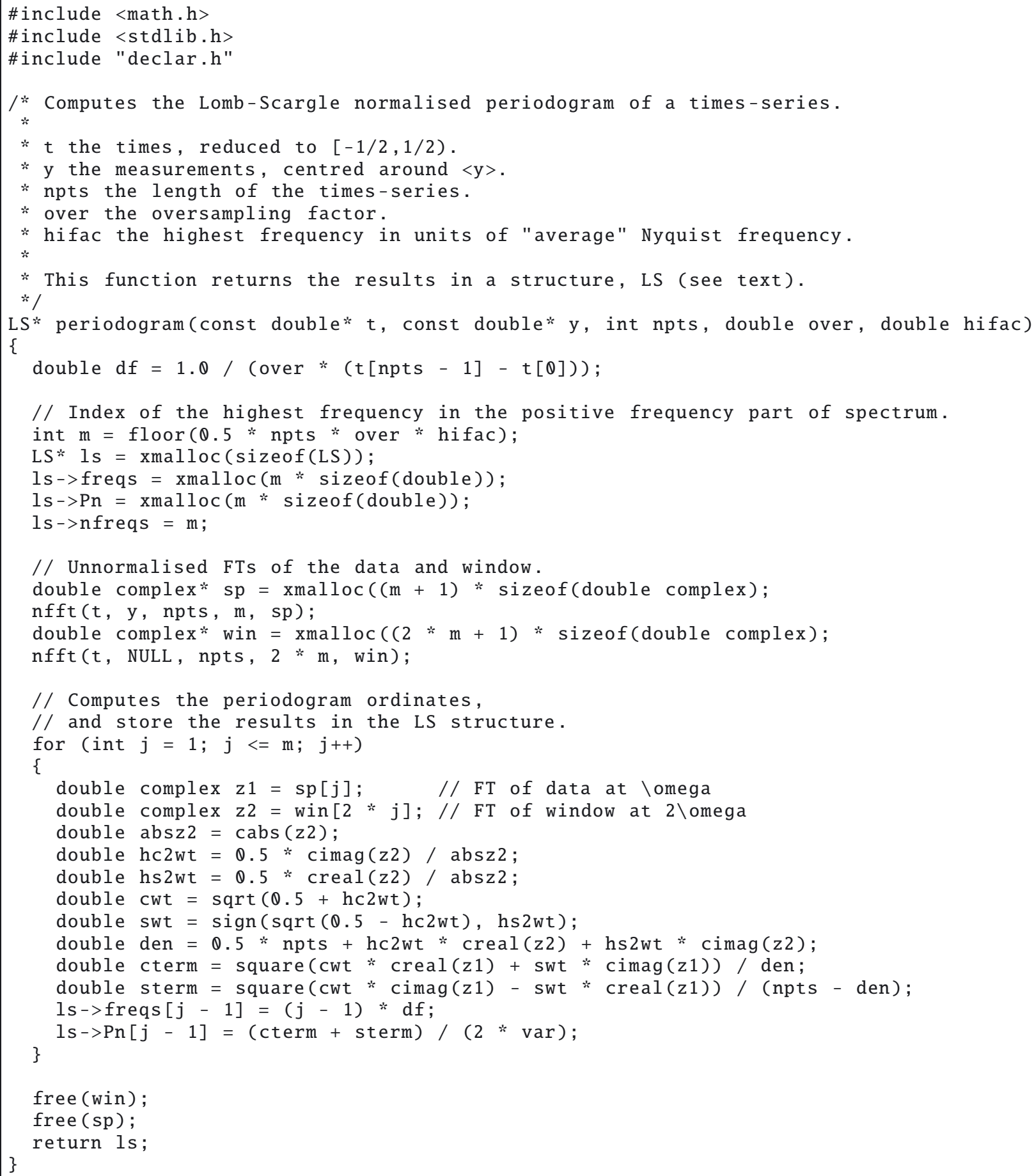

Fig. A.2. Function for computing an NFFT-based LS periodogram.

In practice, simple functions such as sign and square are inlined in the code.

We note that the function periodogram differs from the function fasper from Numerical Recipes (Press et al. 1992) in two important ways. Firstly, the sums of the trigonometric functions at $2 \omega$ in Eq. (7) are evaluated from Fourier transforms of the observational window of a length twice that of the data (lines 36 and 43). Secondly, and more importantly, the Fourier transforms are evaluated as NFFTs (lines 33 and 36). In addition, periodogram does not determine the peak of the maximal height in the periodogram and the corresponding false-alarm probability. In the spirit of the $\mathrm{C}$ language, this is instead delegated to another function (not displayed since it is a trivial translation of Eq. (6)).

\section{References}

Belkacem, K., Samadi, R., Goupil, M.-J., et al. 2009, Science, 324, 1540 Fourmont, K. 1999, Ph.D. Thesis, Mathematisch-Naturwissenschaftlichen Fakulät der Westfälischen Wilhelms-Universität Münster, Germany 
B. Leroy: Lomb-Scargle periodogram using NFFTs

Frescura, F. A. M., Engelbrecht, C. A., \& Frank, B. S. 2008, MNRAS, 388, 1693 Frigo, M., \& Johnson, S. G. 2005, Proc. IEEE, 93,216

Gottlieb, E. W., Wright, E. L., \& Liller, W. 1975, ApJ, 195, L33

Horne, J. H., \& Baliunas, S. L. 1986, ApJ, 302, 757

Jackson, J. I., Meyer, C. H., Nishimura, D. G., \& Macovski, A. 1991, IEEE Trans. Med. Imag., 10, 473

Keiner, J., Kunis, S., \& Potts, D. 2009, ACM Trans. Math. Softw., 36, 1

Koch, D. G., Borucki, W. J., Basri, G., et al. 2010, ApJ, 713, L79

Lomb, N. R. 1976, Ap\&SS, 39, 447

Michel, E., Baglin, A., Weiss, W. W., et al. 2008, Commun. Asteroseismol., 156, 73

NIST 2011, Digital Library of Mathematical Functions, National Institute of Standards and Technology, Release date: 2011-08-29,

http://dlmf.nist.gov/

Pippig, M. 2009, Ph.D. Thesis, Chemnitz University of Technology

Potts, D., Steidl, G., \& Tasche, M. 1998, in Modern Sampling Theory: Mathematics and Applications, eds. J. Benedetto, \& P. Ferreira, 249
Press, W. H., \& Rybicki, G. B. 1989, ApJ, 338, 277

Press, W. H., Tukolsky, S. A., Vetterling, W. T., \& Flannery, B. P. 1992, Numerical Recipes in C: The Art of Scientific Computing, 2nd edn (Cambridge University Press)

Reegen, P. 2007, A\&A, 467, 1353

Scargle, J. D. 1982, ApJ, 263, 835

Scargle, J. D. 1989, ApJ, 343, 874

Schaback, R. 1995, in Approximation Theory VIII, Vol. 1: Approximation and Interpolation, eds. C. Chui, \& L. Schumaker (World Scientific Publishing Co., Inc.), 491

Schwarzenberg-Czerny, A. 1998, MNRAS, 301, 831

Sørensen, T. S., Schaeffer, T., Noe, K. O., \& Hansen, M. 2008, IEEE Trans. Med. Imag., 27, 538

Steidl, G. 1998, Adv. Comput. Math., 9, 337

Townsend, R. H. D. 2010, ApJS, 191, 247

Waelkens, C., Aerts, C., Kestens, E., Grenon, M., \& Eyer, L. 1998, A\&A, 330, 215 